Research Article

Human and Medical Genetics

\title{
Integrated analysis of IncRNA-associated ceRNA network identified potential regulatory interactions in osteosarcoma
}

\author{
Yongwei Wang ${ }^{1}$, Yaxian Gao ${ }^{2}$ iD, Sen Guo ${ }^{1}$ and Zhihong Chen ${ }^{1}$ \\ ${ }^{1}$ Department of Anatomy, Basic Medical Institute, Chengde Medical College, Chengde 067000, Hebei, China. \\ ${ }^{2}$ Department of Immunology, Basic Medical Institute, Chengde Medical College, Chengde 067000, Hebei, China.
}

\begin{abstract}
This study aimed to identify potential therapeutic targets in osteosarcoma (OS) through the network analysis of competing endogenous RNAs (ceRNAs). The differentially expressed miRNAs (DEMIs) and mRNAs (DEMs) were identified between OS cell lines and human mesenchymal stem cells (hMSCs) from the data deposited under GSE70415 using limma package. Functional analysis of DEMs was performed using DAVID and clusterProfiler to identify significantly enriched Gene Ontology biological processes and KEGG pathways, respectively. The DEMI-DEM interaction network was constructed using Cytoscape. LncRNA-miRNA interactions were predicted using starBase database. The ceRNA regulatory network was constructed by integrating mRNAs, miRNAs, and IncRNAs, and functional enrichment analysis was performed for the genes involved. The analysis revealed a total of 326 DEMs and 54 DEMls between OS cells and hMSCs. We identified several novel therapeutic targets involved in the progression and metastasis of OS, such as CBX7, RAD9A, SNHG7 and miR-34a-5p. The miRNA, miR-543 (target gene: CBX7) was found to be associated with the pathway Mucin type O-glycan biosynthesis. Using the ceRNA network, we established the following regulatory interactions: NEAT1/miR-543/CBX7, SNHG7/miR-34a-5p/RAD9A, and XIST/miR-34a-5p/RAD9A. CBX7, RAD9A, IncRNA SNHG7, miR-543, and miR-34a-5p may be explored as novel therapeutic targets for treatment of OS.
\end{abstract}

Keywords: Osteosarcoma, differentially expression mRNA, differentially expression miRNA, competing endogenous RNAs network, lncRNA.

Received: March 15, 2019; Accepted: March 05, 2020.

\section{Introduction}

Osteosarcoma (OS) is the most commonly diagnosed primary malignant bone tumor in adolescents (He et al., 2014). The age-adjusted worldwide incidences per million of OS in the age group of 0-24 is 4.4 , with the incidence rate in males being more than females in every race (male:female $=1.34: 1)$ (Mirabello et al., 2009). Though the 5-year overall survival rate for OS is 68\% (Ottaviani and Jaffe, 2009), it is highly metastatic (Marina et al., 2004), with lung being the most common site of metastasis (Fitzgerald et al., 1973). The complex molecular mechanisms associated with progression and metastasis of OS is not clear and is challenging to treat. Hence, it is important to identify the underlying molecular mechanisms associated with the development and metastasis of OS and identify better therapeutic targets for its treatment.

Long non-coding RNAs (lncRNAs), a class of nonprotein-coding RNA transcripts longer than 200 nucleotides (Morris and Mattick, 2014), are known to be involved

Send correspondence to: Yaxian Gao. Department of Immunology, Basic Medical Institute, Chengde Medical College, Anyuan Road, Shuangqiao District, Chengde 067000, Hebei, China. E-mail: yaxiangao@163.com. in many biological processes, such as transcriptional regulation, cell proliferation, metastasis and tumorigenesis (Tang et al., 2016; Wei et al., 2016). Earlier studies have implicated that lncRNAs may be important factors in the malignant transformation of the tumors, and can be considered as novel biomarkers (Yarmishyn and Kurochkin, 2015). Similarly, micro RNAs (miRNAs), a class of short non-coding RNAs, play important regulatory roles in many biological processes, such as cell migration, apoptosis, cell differentiation and oncogenesis, by targeting the mRNA molecules (Wang et al., 2015). It is well known that lncRNAs act as competing endogenous RNA (ceRNA) and affect the level of mRNAs by negatively regulating miRNA expression (Salmena et al., 2011; Liu et al., 2014; Cao et al., 2015; Wang et al., 2016). For example, the lncRNA TUG1 acts as a ceRNA for miR-335-5p and promotes OS cells migration and invasion (Yong et al., 2017). Uzan et al. reported that high expression of the IncRNA HULC promotes metastasis and is associated with poor prognosis of OS (Uzan et al., 2016). The up-regulated lncRNA $H N F 1 A-A S 1$ promotes proliferation and metastasis of OS cells by activating Wnt/catenin signaling pathway (Zhao $\mathrm{H}$ et al., 2016a). However, the complex mechanisms associ- 
ated with metastasis of OS has not been fully understood. The integrative analysis can help us in better understanding of related genes, functions, and the complex mechanisms associated with the development and metastasis of OS.

The aim of the present study is to understand the molecular mechanisms by exploring the regulatory interactions through ceRNA regulatory network, and identify potential therapeutic targets in OS using bioinformatics analysis.

\section{Material and Methods}

The flow of the analysis steps used in this study is shown in Figure 1.

\section{Data source and preprocessing}

The mRNA and miRNA expression profiles were downloaded from the Gene Expression Omnibus (GEO) series GSE70415 (Wang H et al., 2017a). This series included two subseries: GSE70414 and GSE70367 corresponding to mRNA and miRNA expression data, respectively. The original study used five OS cell lines and $\mathrm{t}$ human mesenchymal stem cells, (hMSCs) as control. The mRNA expression data was generated using Affymetrix Human Genome U133 Plus 2.0 (HG-U133_Plus_2) array (GPL570), and the miRNA expression data was generated using Affymetrix Multispecies miRNA-3 (miRNA-3) array (GPL16384). The original study published by Wang et al. (2017a) corresponding to the data deposited in GSE70415, mainly focused on miRNA-mRNA regulatory interactions, however, the interactions between IncRNAs and miRNAs were studied.

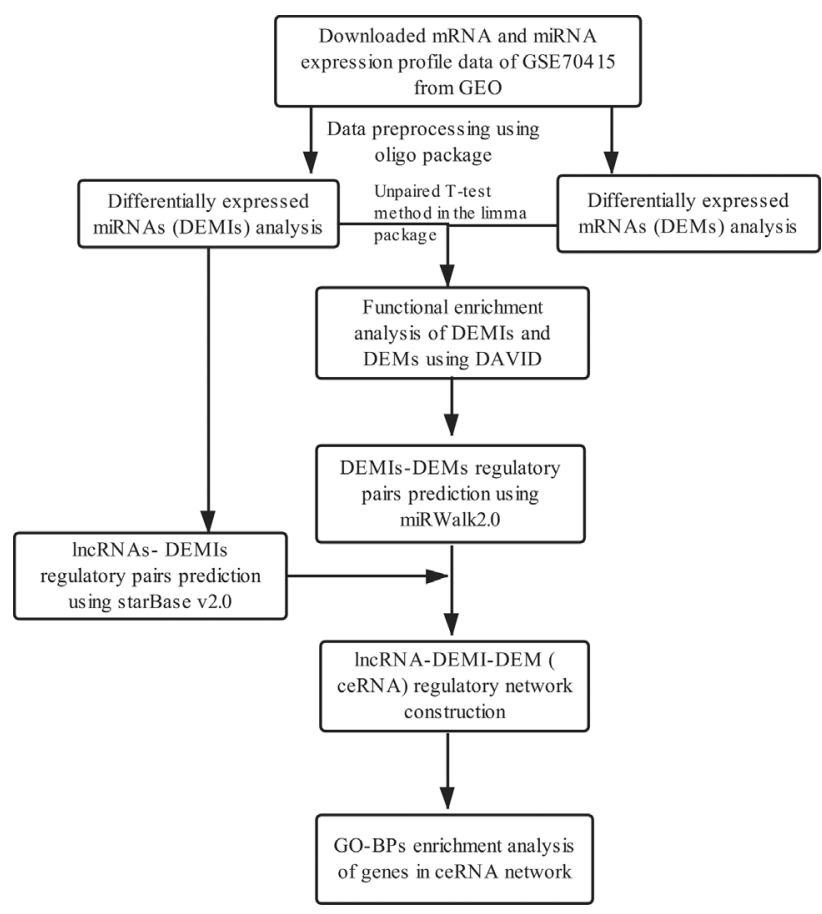

Figure 1 - Overall strategy and analyses followed in the current study.
The R-based oligo package (Irizarry et al., 2003) was used for data preprocessing, including background correction, normalization and summarization (median-polish). The Robust Multichip Average (RMA) method in oligo package was used to implement background correction with treating the perfect-match probe intensities as a convolution of noise and true signal, followed by normalization. The annotation file was used to map the probes in the mRNA matrix to gene symbols. When multiple probes corresponded to the same gene symbol, summarization at the probeset level was performed, and the mean expression values across multiple probe-sets were considered as the final expression value for that gene. Since the miRNA platform was composed of multi-species probes, the expression values of probes corresponding to human miRNA were selected for further analysis.

\section{Identification of differentially expressed mRNAs and miRNAs between OS cell lines and hMSCs}

The differentially expressed miRNAs (DEMIs) and mRNAs (DEMs) were identified using the unpaired T-test method implemented in limma package (Smyth, 2005). The DEMIs and DEMs with a P-value $<0.05$ and $\log 2$ fold change $(\log 2 \mathrm{FC})>1$ were considered as statistically significant. The heatmaps and volcano plots of the significant DEMIs and DEMs were constructed using the R-based pheatmap (Wang et al., 2014) and ggplot2 (Warnes et al., 2009) software packages, respectively.

\section{Regulatory pair prediction and functional enrichment analysis of DEMls and DEMs}

The DEMI probes were annotated using miRBase version 21 to identify the corresponding miRNAs (updated list of miRNAs using miRBase version 22.1 are shown in Table S1). The mRNA targets for the top (based on log2FC) 10 up- and down-regulated DEMIs were predicted using miRWalk2.0 tool (Dweep and Gretz, 2015). Further, the miRNA-target pairs showing consensus across five prediction methods integrated in miRWalk (miRanda, miRDB, miRMap, RNA22, and TargetScan) were considered as the for further analysis. Among the DEMI targets, only those that overlapped with the DEMs were considered for further analysis.

The Kyoto Encyclopedia of Genes and Genomes (KEGG) (Ogata et al., 1999) pathways enriched by the miRNA targeted DEMs were analyzed using the clusterprofiler package in R software (Yu et al., 2012). The KEGG pathways with adjusted $\mathrm{P}$-value $<0.05$ were considered as statistically significant. Database for Annotation, Visualization and Integrated Discovery (DAVID, version 6.8) (Huang et al., 2008) was used to obtain enriched Gene Ontology biological processes (GO-BPs) (Ashburner et al., 2000) for the DEMs targeted by the DEMIs. The GO-BP terms with a gene count $\geq 2$ and $\mathrm{P}$-value $<0.05$ were considered as significantly enriched. 


\section{Construction of DEMI-DEM network}

The regulatory network between DEMIs and DEMs was constructed by using the Cytoscape software, version 3.2.0 (Shannon et al., 2003). The topological properties of the network were analyzed, and the results were presented with the Degree Centrality (DC). The nodes with higher DC scores were considered as hub proteins.

Construction and functional enrichment analysis of genes involved in IncRNA-miRNA-mRNA regulatory network

starBase database provides the most comprehensive CLIP-Seq experimentally supported lncRNA-miRNA interactions. It contains 35,459 human miRNA-lncRNA interactions ( $\mathrm{Li}$ et al., 2014). The regulatory interactions between the lncRNAs and differentially expressed miRNAs were extracted from the starBase database based on the following criteria: medium stringency $\geq 2$; number of cancer types $\geq 1$; clade: mammal; genome: Human; assembly: hg19.

Using the IncRNA-DEMI and DEMI-DEM regulatory interactions, the lncRNA and DEM that were regulated by the same DEMI were identified, and were used for the construction of lncRNA-DEMI-DEM (lncRNA-miRNAmRNA) regulatory network, also known as ceRNA regulatory network.

The enrichment analysis of the genes involved in ceRNA network was performed to obtain GO-BP and annotations with a $\mathrm{P}$-value $<0.05$ and gene count $\geq 2$ were considered for further analysis.

\section{Results}

Identification of DEMs and DEMIs between OS cell lines and hMSCs

The DEMs and DEMIs between OS cell lines and hMSCs were derived using the microarray dataset GSE70414. A total of 326 DEMs (Table S2), including 76 up- and 250 down-regulated were identified, and are shown in the double-level clustering heatmap (Figure 2A) and volcano plot (Figure 2B). The heatmap shows independent clustering of OS cell lines and hMSCs. Similarly, a total of 54 DEMIs, including 26 up- and 28 down-regulated (Figure
2C \& D) were identified. The complete list of DEMs and DEMIs can be found in Table S1 and Table S3, respectively.

\section{Functional enrichment analysis of DEMs and DEMIs}

Functional enrichment analysis was performed to obtain GO-BPs and pathways influencing the development of OS. As shown in Figure 3A, a total of six miRNAs were found to be significantly enriched in 18 KEGG pathways. For example, has-miR-543 was found to be associated with "Mucin type O-glycan biosynthesis" pathway. The GO-BP enrichment analysis showed RPS6KA5 to be significantly related to "in utero embryonic development (GO: $0001701)$ ", EDNRA to be significantly related to "negative regulation of transcription, DNA-templated (GO: 0045892)" and TNFSF4 to be significantly related to "regulation of inflammatory response (GO: 0050727)", Figure 3B.

\section{Analysis of DEMI-DEM regulatory network}

For the 54 DEMIs obtained from the microarray analysis, potential target genes consistent across multiple target prediction tools. The interactions between the target DEMIs and DEMs were used to construct a miRNA-gene regulatory network (e.g., has-miR-543-CBX7, miR-34a5p-RAD9A and has-miR-495-3p-DGKD. The DEMIDEM regulatory network (Figure 4), had a total of 139 nodes and 238 interactions (edges). Top five miRNAs (miR-495-3p, miR-543, miR-34a-5p, miR-182-5p and miR-760) and genes (EPG5, ADAMTSL1, CDC42EP3, NEDD4L, and $P T G S 1$ ) identified based on the degree ranking are shown in Table 1.

\section{Analysis of ceRNA regulatory network}

To obtain miRNA and lncRNA interactions, the lncRNAs regulating 19 miRNAs present in the DEMIDEM network were predicted (Supplemental File S1). The miRNA-lncRNA interaction network consisted of 16 nodes, including seven miRNAs and nine lncRNAs. Examples of lncRNA-miRNA interactions include NEAT1-hasmiR-543, SNHG7-has-miR-34a, and XIST-miR-34a-5p. Finally, the lncRNA-miRNA and miRNA-gene regulatory networks were merged to obtain the integrated ceRNA regulatory network. The ceRNA network had 145 pair-wise in-

Table 1 - The top 5 differentially expressed miRNAs and differentially expressed genes between osteosarcoma cells and human mesenchymal stem cells

\begin{tabular}{|c|c|c|c|c|c|}
\hline miRNA & Description & Degree & Gene & Description & Degree \\
\hline hsa-miR-495-3p & down & 29 & EPG5 & down & 6 \\
\hline hsa-miR-543 & down & 25 & $N E D D 4 L$ & up & 5 \\
\hline hsa-miR-34a-5p & down & 24 & ADAMTSL1 & down & 5 \\
\hline hsa-miR-182-5p & up & 21 & CDC42EP3 & down & 5 \\
\hline hsa-miR-760 & up & 19 & PTGS1 & down & 5 \\
\hline
\end{tabular}

miRNAs, microRNAs. 

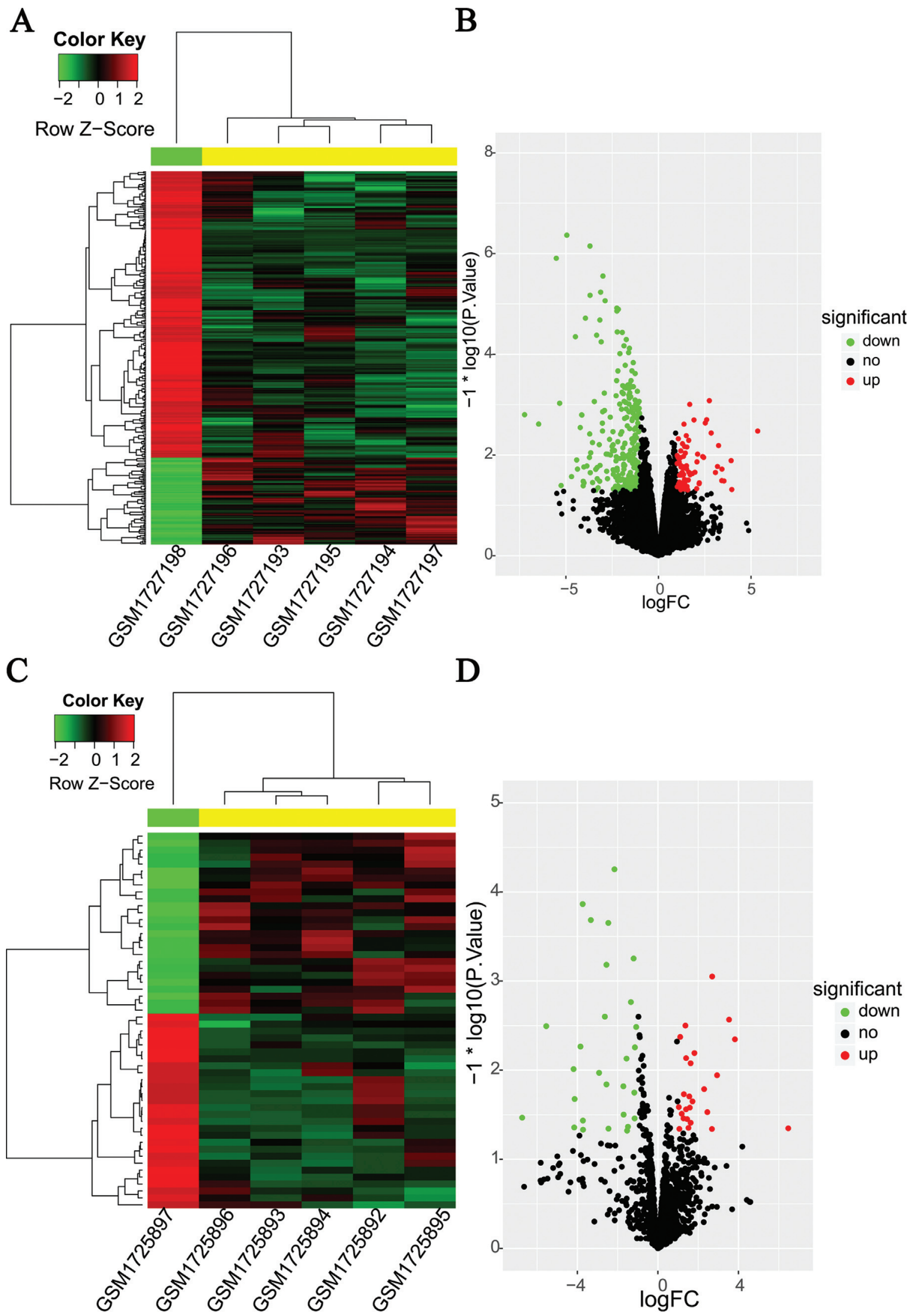

Figure 2 - Heatmap and volcano plot of differentially expressed mRNAs (A \& B, respectively) and miRNAs (C \& D, respectively) between osteosarcoma (OS) cell lines and human mesenchymal stem cells (hMSCs). In the heatmap, the red color indicates up-regulation, and the green color indicates down-regulation. 

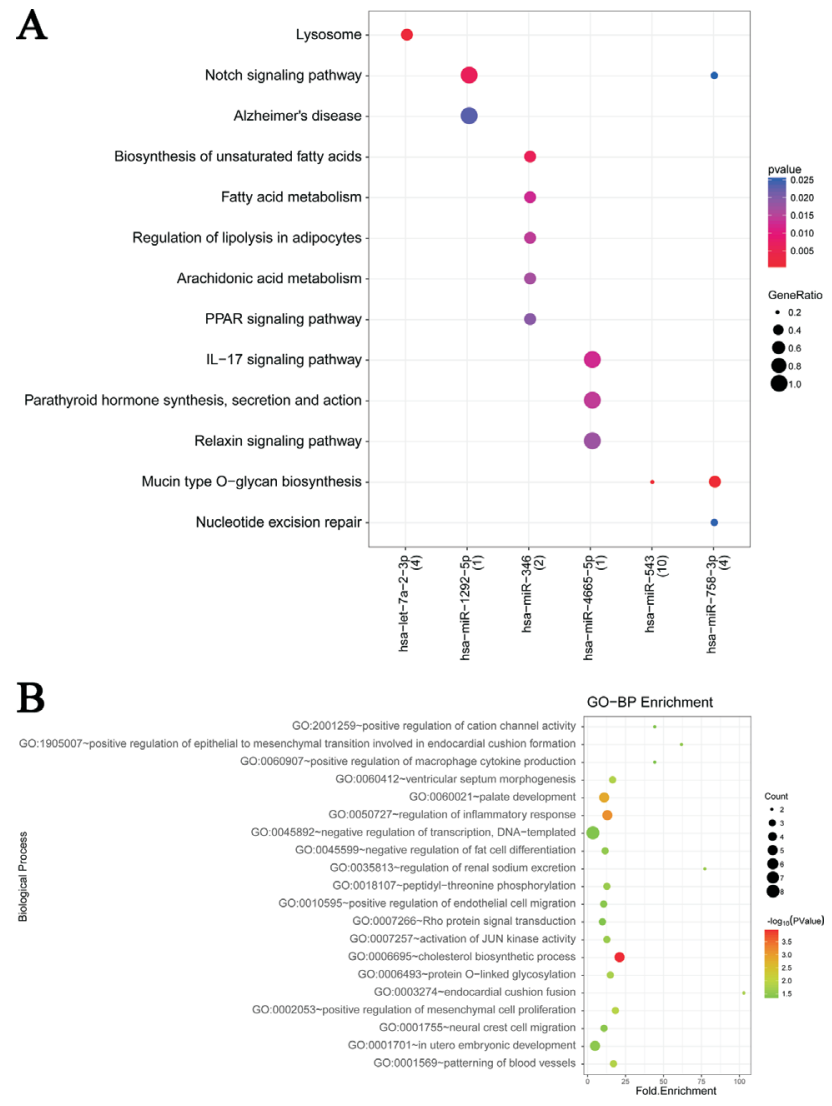

Figure 3 - Functional enrichment analysis of differentially expressed mRNAs and miRNAs between osteosarcoma (OS) cell lines and human mesenchymal stem cells (hMSCs). A: representative enriched pathways by DEMIs; B: representative enriched biological processes by DEMs.

teractions among 105 nodes, consisting of two up-regulated and five down-regulated miRNAs, 20 up-regulated and 69 down-regulated DEMs, and nine lncRNAs (Figure 5 and Table S4). Top five miRNAs, mRNAs and lncRNAs in the network are listed in Table 2.

Function enrichment analysis of the network identified 20 significant GO-BP annotations. As shown in Figure 6 , the DEMs were found to be significantly related to "positive regulation of transcription, DNA-templated (GO: 0045893)" as well as "negative regulation of transcription, DNA-templated (GO: 0045892)".

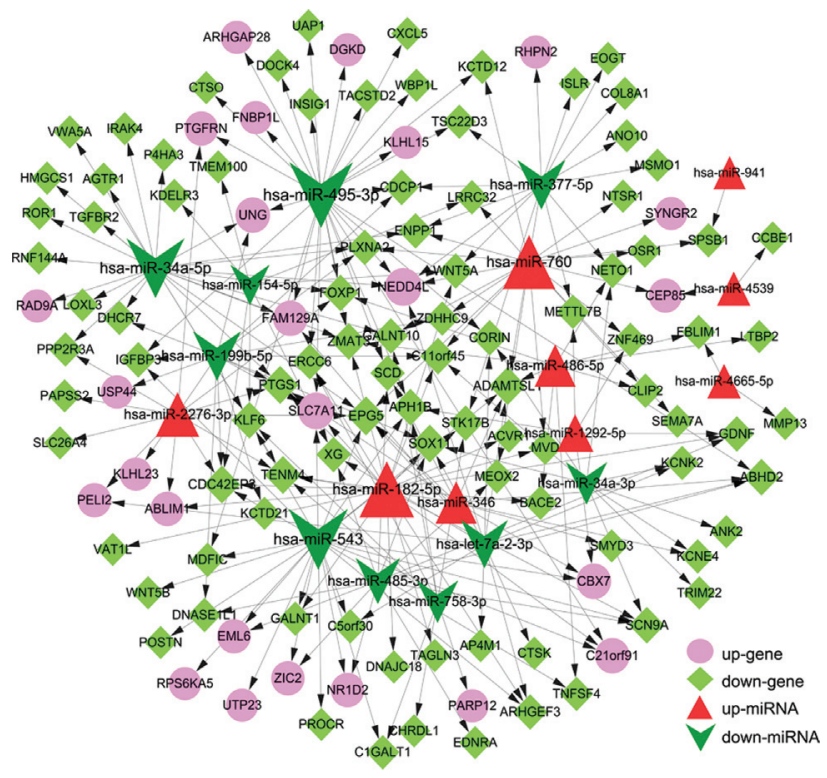

Figure 4 - Regulatory network showing mRNA-miRNA interactions. Pink circles: Up-regulated mRNA;Light green diamonds: Down-regulated mRNA; the Red triangles: Up-regulated miRNA; Green arrows: Down-regulated miRNA.

\section{Discussion}

In the current study, a total of 326 DEMs and 54 DEMIs were identified by re-analyzing the dataset, GSE70415 using limma package. Several potential targets that may be involved in the progression and metastasis of OS were identified, e.g., $C B X 7, \quad R A D 9 A$, SNHG7 (lncRNA) and has-miR-34a-5p. In addition, few potential regulatory interactions, such as NEAT1/ miR-543, miR543/CBX7, SNHG7/ miR-34a-5p, miR-34a-5p/RAD9A, and XIST/miR-34a-5p were identified in the OS cells through the ceRNA network. The dataset GSE70415 had been analyzed in a previous study (Wang H et al., 2017a); and a total of 3,856 DEMs and 250 DEMIs were identified by using GCBI. When compared, the results were found to be similar between the earlier and current study. For example, the key gene POSTN discussed in the earlier study was also found to be highly downregulated in our study. Similarly, the miRNAs, miR-34a, miR-182, miR-493 and miR29 were found to be differentially expressed in the previous

Table 2 - The top 5 miRNAs, lncRNAs, and mRNAs in ceRNA regulatory network

\begin{tabular}{lccccccc}
\hline miRNA & Description & Degree & Gene & Description & Degree & LncRNA & Degree \\
\hline hsa-miR-495-3p & down & 30 & CDC42EP3 & down & 4 & NEAT1 & 3 \\
hsa-miR-34a-5p & down & 28 & SLC7A11 & up & 4 & XIST & SNHG1 \\
hsa-miR-543 & down & 27 & ZMAT3 & down & 4 & SNHG7 & 2 \\
hsa-miR-182-5p & up & 23 & KLF6 & down & 3 & SNH & KCNQ1OT1 \\
hsa-miR-199b-5p & down & 15 & C2lorf91 & up & 3 & KC \\
\hline
\end{tabular}

lncRNA, long non-coding RNA; ceRNA, competing endogenous RNAs. 


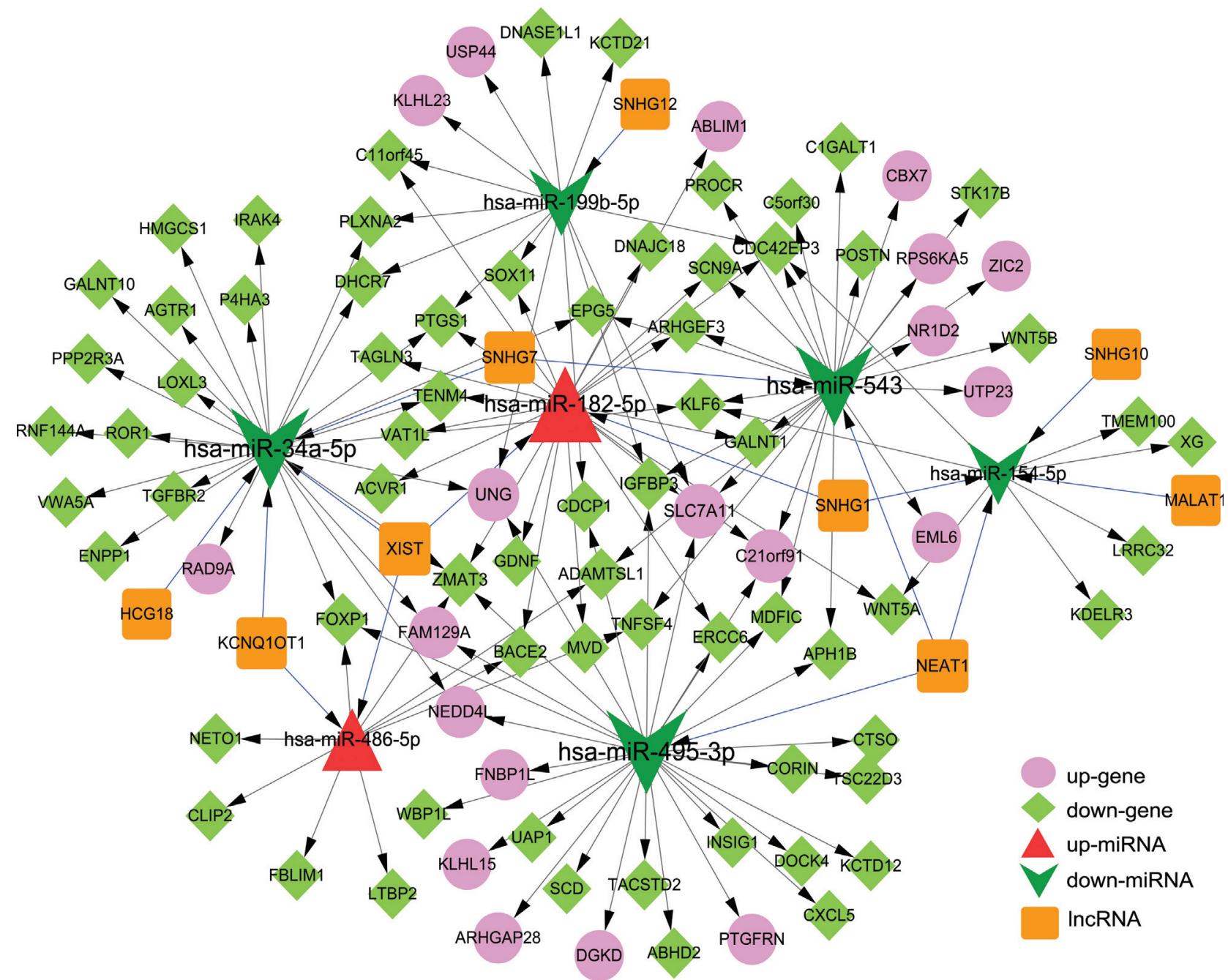

Figure 5 - Competing-endogenous RNA regulatory network. Pink circles: Up-regulated mRNA; Light green diamonds: Down-regulated mRNA; Red triangles: Up-regulated miRNA; Green arrows: Down-regulated miRNA; Orange squares: LncRNA. Blue lines (edges): LncRNA-miRNA interactions; Gray lines (edges): miRNA-mRNA interactions.

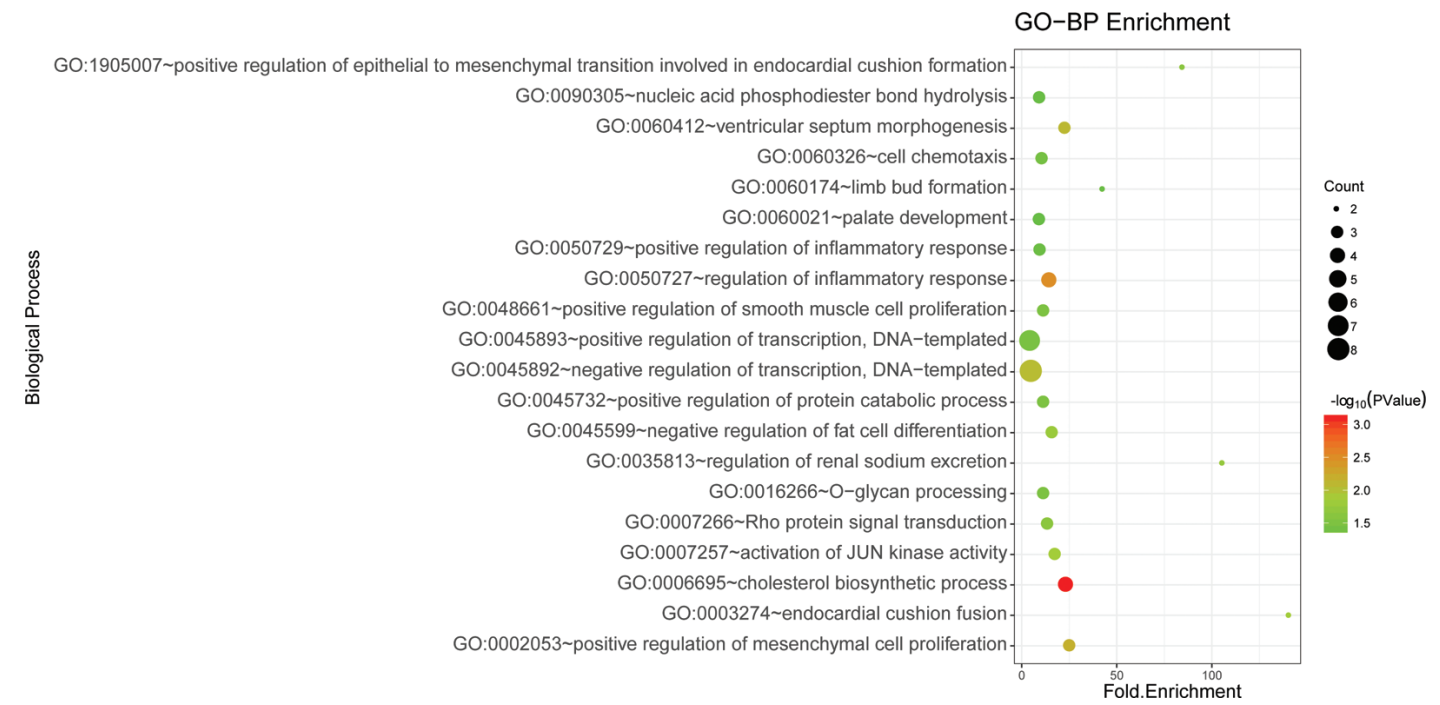

Figure 6 - Enrichment of Gene ontology biological processes by differentially expressed mRNAs involved in the ceRNA regulatory network. Count represents the number of genes associated with the annotation 
study. However, there were significant differences observed between the current and the previous study. For instance, the GO annotations including extracellular matrix organization, small molecule metabolic process, cell adhesion, and KEGG pathways including PI3K-Akt signaling pathway and metabolic pathways were found to be significantly enriched in the previous study, however not in the current study. One of the reasons behind this could be the method used for analysis. Additionally, we performed IncRNA and ceRNA network analysis, which was not performed by the previous study.

Nuclear enriched abundant transcript 1 (NEAT1) is a lncRNA transcribed from the multiple endocrine neoplasia locus. Study by Zhao et al., have shown that NEATl acts as an oncogene and is associated with the development of OS (Zhao H et al., 2016b). Knockdown of NEAT1 is known to inhibit tumor cells proliferation and metastases and induce cell apoptosis (Wang $\mathrm{H}$ et al., 2017b). In addition, it promotes oncogenic proliferation by affecting the epigenetic structure of target gene promoters and driving their transcription in prostate cancer (Chakravarty et al., 2014). In the current study, miR-543 was predicted to be a target of NEAT1. The molecular mechanism of this interaction has not been characterized in the progression of OS. The expression of miR-543 inhibits epithelial-mesenchymal transition during tumor metastasis (Haga and Phinney, 2012). Over-expression of miR-543 inhibits tumor cell proliferation and has been reported to reduce the migration and invasion of tumor cells in endometrial cancer (Bing et al., 2014). Based on the published studies and findings from the current study, it can be hypothesized that the lncRNA NEAT1 promotes proliferation and metastasis of OS cells by sponging the miRNA, miR-543.

Chromobox 7 ( $C B X 7$, also known as chromobox homolog 7), a polycomb family protein, and a component of the polycomb repressive complex 1 , is known to extend the lifespan of some normal human cells. Reportedly, the over-expressed $C B X 7$ acts as an oncogene in the gastric cancer (Zhang et al., 2010). Further, studies have shown high expression of $C B X 7$ in various prostate cancer cell lines (Bernard et al., 2005) and clear cell adenocarcinoma of the ovary (Shinjo et al., 2014). In addition, another member of the polycomb family, chromobox 4 ( $C B X 4$, also known as chromobox homolog 4) is known to be involved in progression of OS, and over-expression of $C B X 4$ is associated with advanced clinical stage of OS (Yang et al., 2016). However, the role of $C B X 7$ in OS progression has been not reported. Based on the existing evidences for $C B X 4$, we presume that $C B X 7$ may act as an oncogene during the development of OS. In addition, based on IncRNA regulatory interactions, we believe that the IncRNA NEAT1 may affect the expression of $C B X 7$ by competing for miR543 during the progression of OS.

Small nucleolar RNA host gene 7 (SNHG7) belongs to the long non-coding RNA class. Reportedly, SNHG7 contributes to the growth and metastasis of glioblastoma by suppressing miR-5059 and activating the wnt/ $\beta$-catenin signaling pathway (Ren et al., 2018). Additionally, in prostate cancer, $S N H G 7$ promotes cell proliferation via cyclin D1 by modulating miR-503 (Qi et al., 2018). Further, $S N H G 7$ sponges miR-34a and induces over-expression of GALNT7, which in turn results in the progression of colorectal cancer (Li et al., 2018). In our study, miR-34a-5p was predicted to be a target of $S N H G 7$, for the first time in OS cell lines. It was reported as a tumor suppressor in for the first time in neuroblastoma (Welch et al., 2007). The overexpression of miR-34a has been reported to inhibit cell growth and induce differentiation of glioma stem cells in human glioma tumors (Guessous et al., 2010). The, lncRNA C2dat 1 has been shown to promote cell proliferation, metastasis and infiltration of OS cells by targeting miR-34a-5p (Jia et al., 2018). Thus, our results suggest that lncRNA $S N H G 7$ may promote cell proliferation and metastasis of OS cells by suppressing miR-34a-5p.

RAD9 checkpoint clamp component A ( $R A D 9 A$, also known as $R A D 9$ ) is a cell cycle checkpoint protein associated with cell cycle arrest and DNA damage repair. It is an oncogene and is known to be regulated by DNA methylation, and its chromosome locus 11q13 has been reported to be amplified in breast cancer (Cheng et al., 2005). A study by Lieberman et al., has shown abnormal over-expression of $R A D 9$ in approximately $45 \%$ of clinically detected prostate tumors, and its significant correlation with tumor stage (Lieberman et al., 2018). However, the role of RAD9A in OS is has not been explored. In the current study, RAD9A was predicted to be a target of miR-34a-5p. A study by Wang et al. has shown that over-expression of miR-34a-5p inhibits cell proliferation and metastasis of cervical cancer cells and promotes cell apoptosis (Wang X et al., 2017c). In colon cancer cells (HCT116), the expression of miR-34a$5 \mathrm{p}$ has been reported to induce apoptosis, cell cycle arrest at G1 stage and transcription of P53 (Gao et al., 2015). Hence, based on previous reports and the results from the current study, it can be implicated that the RAD9A regulation by miR-34a-5p may affect the progression of OS through cell cycle regulation. In summary, the IncRNA $S N H G 7$ and the protein coding gene $R A D 9 A$ may play an important role in the progression of OS by competing with each other for miR-34a-5p.

$\mathrm{X}$ inactivate-specific transcript (XIST) is a wellknown lncRNA. The high expression of XIST is associated with cell proliferation and poor prognosis of OS ( $\mathrm{Li}$ et al., 2017). In our ceRNA network, miR-34a-5p was predicted to be a target of XIST. This regulatory interaction has been reported in multiple cancers, such as pancreatic cancer (Sun Z et al., 2018), colon cancer (Sun N et al., 2018), and human nasopharyngeal carcinoma (Song et al., 2016). However, the underlying regulatory mechanisms of XIST have been hardly reported in the progression of OS. The current study indicates that interaction of XIST and miR-34a-5p 
may regulate downstream mRNA levels associated with progression of OS.

The interaction of different RNA molecules explored in the current study may provide insights into novel targets and underlying molecular mechanisms associated with development and metastasis of OS. However, the current study has limitations. The results obtained in the current study including, genes and their interactions need to be validated using in vivo and in vitro experimental approaches to confirm their regulatory roles. Further, the number of samples considered by the dataset used in the current study is less.

In conclusion, $C B X 7, R A D 9 A, S N H G 7$ lncRNA and has-miR-34a-5p may be explored as novel therapeutic targets for the treatment of OS. Further, the lncRNA NEAT1 may be involved in promoting the proliferation and metastasis of OS cells by sponging miR-543. The IncRNA $S N H G 7$ and the protein coding gene $R A D 9 A$ may play a role in the progression of OS through their competitive interaction with miR-34a-5p. Finally, the interaction between the IncRNA XIST and miR-34a-5p may be important in understanding molecular mechanisms associated with OS.

\section{Acknowledgments}

This work was supported by the Science and Technology Research Project for Colleges and Universities in Hebei Province (grant number: QN2016060, QN2015044) and Medical Scientific Research Project for Hebei Provincial Health and Family Planning Commission (grant number: 20160313, 20160310).

\section{Conflict of Interest}

The authors declare that there is no conflict of interest that could be perceived as prejudicial to the impartiality of the reported research.

\section{Author Contributions}

YW conceived and designed the study, obtained funding, and drafted and revised the manuscript; YG acquired the data; SG analyzed and interpreted the data; ZC performed the statistical analysis; all authors have read and approved the final version of the manuscript.

\section{References}

Ashburner M, Ball CA, Blake JA, Botstein D, Butler H, Cherry JM, Davis AP, Dolinski K, Dwight SS, Eppig JT et al. (2000) Gene Ontology: tool for the unification of biology. Nat Genet 25:25-29.

Bernard D, Martinezleal JF, Rizzo S, Martinez D, Hudson D, Visakorpi T, Peters G, Carnero A, Beach D, and Gil J (2005) CBX7 controls the growth of normal and tumor-derived prostate cells by repressing the Ink4a/Arf locus. Oncogene 24:5543-5551.
Bing L, Hong C, Li-Xin S and Wei G (2014) MicroRNA-543 suppresses endometrial cancer oncogenicity via targeting FAK and TWIST1 expression. Arch Gynecol Obstet 290:533541.

Cao SE, Tian J, Chen S, Zhang X and Zhang Y (2015) Role of miR-34c in ketamine-induced neurotoxicity in neonatal mice hippocampus. Cell Biol Int 39:164-168.

Chakravarty D, Sboner A, Nair SS, Giannopoulou E, Li R, Hennig S, Mosquera JM, Pauwels J, Park K, Kossai M et al. (2014) The oestrogen receptor alpha-regulated lncRNA NEAT1 is a critical modulator of prostate cancer. Nat Commun 5:5383.

Cheng CK, Chow LW, Loo WT, Chan TK and Chan V (2005) The cell cycle checkpoint gene Rad9 is a novel oncogene activated by 11q13 amplification and DNA methylation in breast cancer. Cancer Research 65:8646-8654.

Dweep H and Gretz N (2015) miRWalk2.0: a comprehensive atlas of microRNA-target interactions. Nat Methods 12:697.

Fitzgerald RJ, Dahlin D and Sim F (1973) Multiple metachronous osteogenic sarcoma. Report of twelve cases with two longterm survivors. J Bone Joint Surg Am 55:595-605.

Gao J, Li N, Dong Y, Li S, Xu L, Li X, Li Y, Li Z, Ng SS, Sung JJ et al. (2015) miR-34a-5p suppresses colorectal cancer metastasis and predicts recurrence in patients with stage II/III colorectal cancer. Oncogene 34:4142-52.

Guessous F, Zhang Y, Kofman A, Catania A, Li Y, Schiff D, Purow B and Abounader R (2010) microRNA-34a is tumor suppressive in brain tumors and glioma stem cells. Cell Cycle 9:1031-1036.

Haga CL and Phinney DG (2012) MicroRNAs in the imprinted DLK1-DIO3 region repress the epithelial-to-mesenchymal transition by targeting the TWIST1 protein signaling network. J Biol Chem 287:42695-707.

He JP, Hao Y, Wang XL, Yang XJ, Shao JF, Guo FJ and Feng JX (2014) Review of the molecular pathogenesis of osteosarcoma. Asian Pac J Cancer Prev 15:5967-5976.

Huang da W, Sherman BT and Lempicki RA (2008) Systematic and integrative analysis of large gene lists using DAVID bioinformatics resources. Nat Protoc 4:44-57.

Irizarry RA, Hobbs B, Collin F, Beazer-Barclay YD, Antonellis KJ, Scherf U and Speed TP (2003) Exploration, normalization, and summaries of high density oligonucleotide array probe level data. Biostatistics 4:249-264.

Jia D, Niu Y, Li D and Liu Z (2018) LncRNA C2dat1 promotes cell proliferation, migration, and Invasion by targeting MiR-34a-5p in osteosarcoma cells. Oncol Res 26:753-764.

Li GL, Wu YX, Li YM and Li J (2017) High expression of long non-coding RNA XIST in osteosarcoma is associated with cell proliferation and poor prognosis. Eur Rev Med Pharmacol Sci 21:2829-2834.

Li W, Ma H and Sun J (2014) MicroRNA-34a/c function as tumor suppressors in Hep-2 laryngeal carcinoma cells and may reduce GALNT7 expression. Mol Med Rep 9:1293-1298.

Li Y, Zeng C, Hu J, Pan Y, Shan Y, Liu B and Jia L (2018) Long non-coding RNA-SNHG7 acts as a target of miR-34a to increase GALNT7 level and regulate PI3K/Akt/mTOR pathway in colorectal cancer progression. J Hematol Oncol 11:89.

Lieberman HB, Rai AJ, Friedman RA, Hopkins KM and Broustas CG (2018) Prostate cancer: unmet clinical needs and RAD9 as a candidate biomarker for patient management. Transl Cancer Res 7 Suppl 6:S651-S661. 
Liu XH, Sun M, Nie FQ, Ge YB, Zhang EB, Yin DD, Kong R, Xia $\mathrm{R}, \mathrm{Lu} \mathrm{KH}$, Li JH et al. (2014) Lnc RNA HOTAIR functions as a competing endogenous RNA to regulate HER2 expression by sponging miR-331-3p in gastric cancer. Mol Cancer 13:92.

Marina N, Gebhardt M, Teot L and Gorlick R (2004) Biology and therapeutic advances for pediatric osteosarcoma. Oncologist 9:422-41.

Mirabello L, Troisi RJ and Savage SA (2009) Osteosarcoma incidence and survival rates from 1973 to 2004: data from the Surveillance, Epidemiology, and End Results Program. Cancer 115:1531-1543.

Morris KV and Mattick JS (2014) The rise of regulatory RNA. Nat Rev Genet 15:423-437.

Ogata H, Goto S, Sato K, Fujibuchi W, Bono H and Kanehisa M (1999) KEGG: Kyoto Encyclopedia of Genes and Genomes. Nucleic Acids Research 27:29-34.

Ottaviani G and Jaffe N (2009) The epidemiology of osteosarcoma. Cancer Treat Res 152:3-13.

Qi H, Wen B, Wu Q, Cheng W, Lou J, Wei J, Huang J, Yao X and Weng G (2018) Long noncoding RNA SNHG7 accelerates prostate cancer proliferation and cycle progression through cyclin D1 by sponging miR-503. Biomed Pharmacother 102:326-332.

Ren J, Yang Y, Xue J, Xi Z, Hu L, Pan SJ and Sun Q (2018) Long noncoding RNA SNHG7 promotes the progression and growth of glioblastoma via inhibition of miR-5095. Biochem Biophys Res Commun 496:712-718.

Salmena L, Poliseno L, Tay Y, Kats L and Pandolfi PP (2011) A ceRNA hypothesis: the Rosetta Stone of a hidden RNA language? Cell 146:353-358.

Shannon P, Markiel A, Ozier O, Baliga NS, Wang JT, Ramage D, Amin N, Schwikowski B and Ideker T (2003) Cytoscape: A software environment for integrated models of biomolecular interaction networks. Genome Res 13:2498-2504.

Shinjo K, Yamashita Y, Yamamoto E, Akatsuka S, Uno N, Kamiya A, Niimi K, Sakaguchi Y, Nagasaka T, Takahashi T et al. (2014) Expression of chromobox homolog 7 (CBX7) is associated with poor prognosis in ovarian clear cell adenocarcinoma via TRAIL-induced apoptotic pathway regulation. Int J Cancer 135:308-318.

Smyth GK (2005) Limma: Linear models for microarray data. In: Gentleman R, Carey VJ, Huber W, Irizarry RA and Dudoit S (eds) Bioinformatics and computational biology solutions using R and bioconductor. Springer, New York.

Song P, Ye LF, Zhang C, Peng T and Zhou XH (2016) Long non-coding RNA XIST exerts oncogenic functions in human nasopharyngeal carcinoma by targeting miR-34a-5p. Gene 592:8-14.

Sun N, Zhang G and Liu Y (2018) Long non-coding RNA XIST sponges miR-34a to promotes colon cancer progression via Wnt/ $\beta$-catenin signaling pathway. Gene 665:141-148.

Sun Z, Zhang B and Cui T (2018) Long non-coding RNA XIST exerts oncogenic functions in pancreatic cancer via miR34a-5p. Oncology Reports 39:1591-1600.

Tang Y, Cui Y, Li Z, Jiao Z, Yong Z, Yan H, Chen G, Zhou Q, Wang W, Zhou X et al. (2016) Radiation-induced miR-208a increases the proliferation and radioresistance by targeting p21 in human lung cancer cells. J Exp Clin Cancer Res 35:1-1.
Uzan VR, Lengert Av, Boldrini É, Penna V, Scapulatempo-Neto C, Scrideli CA, Filho AP, Cavalcante CE, de Oliveira CZ, Lopes LF et al. (2016) High expression of HULC is associated with poor prognosis in osteosarcoma patients. Plos One 11:e0156774

Wang H, Tang M, Ou L, Hou M, Feng T, Huang YE, Jin Y, Zhang $\mathrm{H}$ and Zuo G (2017a) Biological analysis of cancer specific microRNAs on function modeling in osteosarcoma. Sci Rep 7:5382.

Wang H, Yu Y, Fan S and Luo L (2017b) Knockdown of long non-coding RNA NEAT1 Inhibits proliferation and invasion and induces apoptosis of osteosarcoma by inhibiting miR194 expression. Yonsei Med J 58:1092-1100.

Wang L, Cao C, Ma Q, Zeng Q, Wang H, Cheng Z, Zhu G, Qi J, Ma H, Nian H et al. (2014) RNA-seq analyses of multiple meristems of soybean: novel and alternative transcripts, evolutionary and functional implications. BMC Plant Biol 14:169-187.

Wang SH, Zhang WJ, Wu XC, Weng MZ, Zhang MD, Cai Q, Zhou D, Wang JD and Quan ZW (2016) The lncRNA MALAT1 functions as a competing endogenous RNA to regulate MCL-1 expression by sponging miR-363-3p in gallbladder cancer. J Cell Mol Med 20:2299-2308.

Wang X, Xie Y and Wang J (2017c) Overexpression of MicroRNA-34a-5p inhibits proliferation and promotes apoptosis of human cervical cancer cells by downregulation of Bcl-2. Oncol Res 26:977-985.

Wang W, Zhang E and Lin C (2015) MicroRNAs in tumor angiogenesis. Life Sci 136:28-35.

Warnes GR, Bolker B, Bonebakker L, Gentleman R, Huber W, Liaw A, Lumley T, Mächler M, Magnusson A, Möller S et al. (2009) gplots: Various R programming tools for plotting data. R Package Version 2:1.

Wei S, Min D, Jiang Z, Hausman GJ, Zhang L and Dodson MV (2016) Long noncoding RNAs in regulating adipogenesis: new RNAs shed lights on obesity. Cell Mol Life Sci 73:1-9.

Welch C, Chen Y and Stallings RL (2007) MicroRNA-34a functions as a potential tumor suppressor by inducing apoptosis in neuroblastoma cells. Oncogene 26:5017-5022.

Yang J, Cheng D, Zhu B, Zhou S, Ying T and Yang Q (2016) Chromobox homolog 4 is positively correlated to tumor growth, survival and activation of HIF- $1 \alpha$ Signaling in human osteosarcoma under normoxic condition. J Cancer 7:427-35.

Yarmishyn AA and Kurochkin IV (2015) Long noncoding RNAs: a potential novel class of cancer biomarkers. Front Genet $6: 145$.

Yong W, Tao Y, Zhen Z, Ming L, Wei Z, Zeng X and Zhang W (2017) Long non-coding RNA TUG1 promotes migration and invasion by acting as a ceRNA of miR-335-5p in osteosarcoma cells. Cancer Sci 108:859-867.

Yu G, Wang LG, Han Y and He QY (2012) clusterProfiler: an R package for comparing biological themes among gene clusters. OMICS 16:284-287.

Zhang XW, Zhang L, Qin W, Yao XH, Zheng LZ, Liu X, Li J and Guo WJ (2010) Oncogenic role of the chromobox protein CBX7 in gastric cancer. J Exp Clin Cancer Res 29:1-7.

Zhao H, Hou W, Tao J, Zhao Y, Wan G, Ma C and Xu H (2016a) Upregulation of lncRNA HNF1A-AS1 promotes cell proliferation and metastasis in osteosarcoma through activation of 
the Wnt/ $\beta$-catenin signaling pathway. Am J Transl Res 8:3503-12.

Zhao H, Zhao Y, Tao J, Ma C, Zhang J, Xu H and Dong Y (2016b) Up-regulated expression of IncRNA NEAT1 promotes progression of osteosarcoma by regulating the activity of Wnt $/ \beta$ catenin pathway. Int J Clin Exp Pathol 9:11466-11472.

\section{Internet Resources}

Gene Expression https:/www.ncbi.nlm.nih.gov/geo/.

Clusterprofiler, http://www.bioconductor.org/packages/release $/ \mathrm{bioc} / \mathrm{html} / \mathrm{clusterProfiler} . \mathrm{html}$.

Database for Annotation, Visualization and Integrated Discovery (DAVID), https://david-d.ncifcrf.gov/starBase, http://starbase.sysu.edu.cn/.

miRBase, http://www.mirbase.org/.

Cytoscape, https://cytoscape.org.
miRWalk2.0, http://zmf.umm.uni-heidelberg.de/apps/zmf/mirwalk2/index.html.

\section{Supplementary material}

Supplemental File S1 - Identifiers and FASTA sequences of lncRNAs

Table S1 - Updated list of miRNAs using miRBase version 22.1

Table S2 - Standardization and annotation mRNA data Table S3 - Standardization and annotation miRNA data Table S4 - Interactions present in the ceRNA network

Associate Editor: Rogerio Margis

License information: This is an open-access article distributed under the terms of the Creative Commons Attribution License (type CC-BY), which permits unrestricted use, distribution and reproduction in any medium, provided the original article is properly cited. 